\title{
A STREAM VIRTUAL ELEMENT FORMULATION OF THE STOKES PROBLEM ON POLYGONAL MESHES*
}

\author{
P. F. ANTONIETTI ${ }^{\dagger}$, L. BEIRÃO DA VEIGA ${ }^{\ddagger}$, D. MORA ${ }^{\S}$, AND M. VERANI $^{\dagger}$
}

\begin{abstract}
In this paper we propose and analyze a novel stream formulation of the virtual element method (VEM) for the solution of the Stokes problem. The new formulation hinges upon the introduction of a suitable stream function space (characterizing the divergence free subspace of discrete velocities) and it is equivalent to the velocity-pressure (inf-sup stable) mimetic scheme presented in [L. Beirão da Veiga et al., J. Comput. Phys., 228 (2009), pp. 7215-7232] (up to a suitable reformulation into the VEM framework). Both schemes are thus stable and linearly convergent but the new method results to be more desirable as it employs much less degrees of freedom and it is based on a positive definite algebraic problem. Several numerical experiments assess the convergence properties of the new method and show its computational advantages with respect to the mimetic one.
\end{abstract}

Key words. virtual elements, mimetic finite differences, Stokes problem, stream function formulation, polygonal meshes

AMS subject classifications. 65N30, 65N12, 76D07, 65N15

DOI. $10.1137 / 13091141 \mathrm{X}$

1. Introduction. Various approaches to extend finite element methods to nontraditional elements (general polygons, pyramids, polyhedra, etc.) have been developed over the last few years; see, e.g., [33, 34, 35, 36]. The construction of basis functions for such elements is a challenging task and may require extensive geometrical analysis. The mimetic finite difference (MFD) method $[16,17,14,5]$ works on general polygonal meshes and preserves the fundamental properties of the underlying physical and mathematical models. Thanks to its great flexibility, the MFD method has been applied successfully to a wide range of problems; see, for instance, [2, 1, 3, 8, 12] and [30] for a much longer list. Very recently, a new evolution of MFD was proposed in [7], taking the name of the virtual element method (VEM). The VEM takes the steps from the main ideas of modern mimetic schemes but follows a Galerkin discretization of the problem, and therefore can be fully interpreted as a generalization of the finite element (FE) method. Thus, the VEM couples the flexilibity of mimetic methods with the theoretical and applicative background of FE methods. Since the VEM are very recent, the present published literature is limited to $[7,18,6,11]$.

A fundamental role in applied problems is represented by the study of reliable and effective numerical methods for fluids. In particular, the simulation of Stokes flows

\footnotetext{
* Received by the editors February 27, 2013; accepted for publication (in revised form) November 6, 2013; published electronically February 12, 2014.

http://www.siam.org/journals/sinum/52-1/91141.html

$\dagger$ MOX-Dipartimento di Matematica, Politecnico di Milano, I-20133 Milano, Italy (paola. antonietti@polimi.it, marco.verani@polimi.it).

${ }^{\ddagger}$ Dipartimento di Matematica, Università di Milano Statale, I-20133 Milano, Italy (lourenco. beirao@unimi.it).

§GIMNAP-Departamento de Matemática, Universidad del Bío-Bío, Concepción, Chile and $\mathrm{CI}^{2} \mathrm{MA}$, Universidad de Concepción, Concepción, Chile (dmora@ubiobio.cl). This author was partially supported by CONICYT-Chile through FONDECYT project 11100180, by DIUBB through project $120808 \mathrm{GI} / \mathrm{EF}$, and Anillo ANANUM, ACT1118, CONICYT (Chile).
} 
(characterized by very small Reynolds number) represents a standalone important problem (e.g., in the context of the numerical simulation of the blood flow) and a crucial step towards the simulation of more complex fluids, such as sedimentation processes. FE methods constitute a classical choice to accomplish this goal; see, e.g., $[22,31,19]$ and the book [27]. A well-established alternative approach is the markerand-cell (MAC) method (see, e.g., [25] and the review [38]), while, among more recent and promising techniques, we mention discrete duality finite volume schemes (see, e.g., $[28,29]$ ). Traditionally, FE methods rely on triangular (simplicial) and quadrilateral meshes. However, in complex simulations one often encounters general polygonal and polyhedral meshes (see, e.g., [33]). In [8, 9, 10] new MFD methods for the Stokes problem on polygonal meshes have been introduced and analyzed. For related works in the context of finite volume methods, see, e.g., [23] and references therein. The connection between the mimetic and finite volume methods have been investigated for the diffusion problem in [24].

In this paper we propose and analyze a novel stream formulation of the VEM for the solution of the Stokes problem. The new formulation hinges upon the introduction of a suitable stream function space (characterizing the divergence free subspace of discrete velocities). We prove that the VEM velocity-pressure scheme of the Stokes problem (which is a reformulation of the MFD method introduced in [8]) is equivalent to our VEM stream formulation. Using general assumptions on the computational domain, we establish that both schemes are well posed and we prove linear convergence for the methods. The computational advantage of the new, equivalent, stream function formulation is twofold. On one side the number of degrees or freedom, being related to a single scalar variable (the stream function) instead of a vector variable (the velocities) plus a scalar one (the pressures), turns out to be much smaller than for the original scheme. Moreover, the ensuing linear system is now positive definite, as opposed to the original one that is undefinite; this allows for more efficient methods such as Cholesky factorization or conjugate gradient. We finally note that, differently from the finite element method where building globally $C^{1}$ functions is complicated, here the stream function formulation can be built with a rather simple construction due to the flexibility of the virtual approach.

The outline of this paper is as follows: In section 2, we discuss the variational formulation of the Stokes problem. In section 3 we recast into the VEM framework the velocity-pressure (inf-sup stable) mimetic scheme presented in [8]. In section 4 we introduce our novel stream formulation and we prove its equivalence with the velocity-pressure virtual element formulation: both schemes are proved to be stable and linearly convergent. Finally, in section 5 we assess the convergence properties of the new stream method and show its computational advantages with respect to the original one based on the velocity pressure formulation.

Throughout the paper, we will follow the usual notation for Sobolev spaces and norms (see, e.g., [20]). In particular, for an open bounded domain $\mathcal{D}$, we will use $|\cdot|_{s, \mathcal{D}}$ and $\|\cdot\|_{s, \mathcal{D}}$ to denote seminorm and norm, respectively, in the Sobolev space $H^{s}(\mathcal{D})$, while $(\cdot, \cdot)_{\mathcal{D}}$ will denote the $L^{2}(\mathcal{D})$ inner product. Often the subscript will be omitted when $\mathcal{D}$ is the computational domain $\Omega$. Moreover, for any subset $\mathcal{D} \subseteq \mathbb{R}^{2}$ and nonnegative integer $k$, we indicate by $\mathbb{P}_{k}(\mathcal{D})$ the space of polynomials of degree up to $k$ defined on $\mathcal{D}$. Finally, $C$ will be a generic constant independent of the decomposition that could change from an occurrence to the other.

2. The Stokes problem. Let $\Omega \subset \mathbb{R}^{2}$ be a simply connected polygonal domain. We consider the two-dimensional Stokes problem 


$$
\left\{\begin{array}{lr}
-\operatorname{div}\left(\nu \boldsymbol{\nabla}^{S} \mathbf{u}\right)-\nabla p=\mathbf{f} & \text { in } \Omega \\
\operatorname{div} \mathbf{u}=0 & \text { in } \Omega \\
\mathbf{u}=\mathbf{0} & \text { on } \partial \Omega
\end{array}\right.
$$

where the symbols div, div $, \nabla, \nabla^{S}$ represent the vector divergence, the divergence, the gradient, and the symmetric gradient operator, respectively. The given external force $\mathbf{f}$ is set in $\left[L^{2}(\Omega)\right]^{2}$, while we assume a constant viscosity $\nu>0$. As usual, the vector field $\mathbf{u}$ represents the velocities and the scalar field $p$ the pressures.

Let $L_{0}^{2}(\Omega)$ denote the space of $L^{2}$ functions with zero average. Introducing the bilinear form $a(\mathbf{u}, \mathbf{v}):=\nu\left(\boldsymbol{\nabla}^{S} \mathbf{u}, \nabla^{S} \mathbf{v}\right)$ the variational formulation of the Stokes problem reads

$$
\left\{\begin{array}{l}
\text { Find } \mathbf{u} \in V:=\left[H_{0}^{1}(\Omega)\right]^{2}, p \in Q:=L_{0}^{2}(\Omega) \text { such that } \\
a(\mathbf{u}, \mathbf{v})+(\operatorname{div} \mathbf{v}, p)=(\mathbf{f}, \mathbf{v}) \quad \forall \mathbf{v} \in V \\
(\operatorname{div} \mathbf{u}, q)=0 \quad \forall q \in Q .
\end{array}\right.
$$

It is well known that problem (2.2) has a unique solution; see, for instance, [27].

Let us introduce the space of divergence-free functions

$$
Z=\{\mathbf{v} \in V: \operatorname{div} \mathbf{v}=0\}
$$

and notice that the solution $\mathbf{u} \in V$ to problem (2.2) is determined by solving the following problem:

$$
\left\{\begin{array}{l}
\text { Find } \mathbf{u} \in Z \text { such that } \\
a(\mathbf{u}, \mathbf{v})=(\mathbf{f}, \mathbf{v}) \quad \forall \mathbf{v} \in Z .
\end{array}\right.
$$

Under the assumption that $\Omega$ is a two-dimensional (2D) simply connected domain, it is well known that for every $\mathbf{v} \in Z$ there exists a uniquely defined scalar potential function $w \in H^{2}(\Omega) / \mathbb{R}$ (see, for instance, [27]) such that

$$
\mathbf{v}=\operatorname{curl} w
$$

where curl $=\left(\frac{\partial}{\partial y},-\frac{\partial}{\partial x}\right)$. Setting

$$
\Phi=\left\{\varphi \in H^{2}(\Omega) / \mathbb{R} \text { such that } \operatorname{curl} \varphi=\mathbf{0} \text { on } \partial \Omega\right\},
$$

we can write the solution $\mathbf{u}$ of (2.3) as $\mathbf{u}=\operatorname{curl} \psi$, where $\psi \in \Phi$ is solution of the following problem:

$$
\left\{\begin{array}{l}
\text { Find } \psi \in \Phi \text { such that } \\
a(\operatorname{curl} \psi, \operatorname{curl} \varphi)=(\mathbf{f}, \operatorname{curl} \varphi) \quad \forall \varphi \in \Phi
\end{array}\right.
$$

We will refer to (2.4) as to the stream function formulation of the Stokes problem $(2.2)$.

3. VEM for Stokes (I): Velocity-pressure formulation. In this section we recast the mimetic finite difference (MFD) method analyzed in $[8,10]$ for the numerical approximation of the Stokes problem into the framework of the VEM recently introduced in [7]. 
Let $\left\{\mathcal{T}_{h}\right\}_{h}$ be a sequence of decompositions of $\Omega$ into elements $K$, let $\mathcal{V}_{h}$ be the set of mesh vertices, let $\mathcal{V}_{h}^{b}$ be the set of boundary vertices, and let $\mathcal{E}_{h}$ be the set of edges $e$ of $\mathcal{T}_{h}$. We assume that for every $h$, the decomposition $\mathcal{T}_{h}$ is made of a finite number of simple polygons (i.e., open simply connected sets with non-selfintersecting boundaries made of a finite number of straight line segments). For all $e \in \mathcal{E}_{h}$ we associate once and for all a normal unit vector $\mathbf{n}_{e}$ and a tangent unit vector $\mathbf{t}_{e}$ obtained by an anticlockwise rotation of $\mathbf{n}_{e}$. Moreover, we denote by $\mathcal{E}_{K}$ the set of edges of $K$.

The bilinear form $a(\cdot, \cdot)$ can obviously be split as

$$
a(\mathbf{u}, \mathbf{v})=\sum_{K \in \mathcal{T}_{h}} a^{K}(\mathbf{u}, \mathbf{v}) \quad \forall \mathbf{u}, \mathbf{v} \in V, \quad a^{K}(\mathbf{u}, \mathbf{v}):=\nu\left(\nabla^{S} \mathbf{u}, \nabla^{S} \mathbf{v}\right)_{K},
$$

with $(\cdot, \cdot)_{K}$ representing the $L^{2}$ scalar product on $K$.

In the following sections, we will show that for each $h>0$ it is possible to build the following:

1. a couple of spaces $\left(V_{h}, Q_{h}\right)$ with $V_{h} \subset V$ and $Q_{h} \subset Q$;

2. a symmetric bilinear form $a_{h}$ from $V_{h} \times V_{h}$ to $\mathbb{R}$ which can be split as

$$
a_{h}\left(\mathbf{u}_{h}, \mathbf{v}_{h}\right)=\sum_{K \in \mathcal{T}_{h}} a_{h}^{K}\left(\mathbf{u}_{h}, \mathbf{v}_{h}\right) \quad \forall \mathbf{u}_{h}, \mathbf{v}_{h} \in V_{h},
$$

where $a_{h}^{K}(\cdot, \cdot)$ is a bilinear form on $V_{h \mid K} \times V_{h \mid K}$;

3. an element $\mathbf{f}_{h} \in V_{h}^{\prime}$ and a discrete duality pair $\langle\cdot, \cdot\rangle_{h}$ in such a way that the resulting discrete problem

$$
\left\{\begin{array}{l}
\text { Find } \mathbf{u}_{h} \in V_{h}, p_{h} \in Q_{h} \text { such that } \\
a_{h}\left(\mathbf{u}_{h}, \mathbf{v}_{h}\right)+\left(\operatorname{div} \mathbf{v}_{h}, p_{h}\right)=\left\langle\mathbf{f}_{h}, \mathbf{v}_{h}\right\rangle_{h} \quad \forall \mathbf{v}_{h} \in V_{h} \\
\left(\operatorname{div} \mathbf{u}_{h}, q_{h}\right)=0 \quad \forall q_{h} \in Q_{h}
\end{array}\right.
$$

has a unique solution $\mathbf{u}_{h}, p_{h}$ and exhibits optimal approximation properties.

3.1. Discrete spaces for velocities and pressures. We first construct the local discrete velocity space $V_{h \mid K}, K \in \mathcal{T}_{h}$. To this aim, we preliminarily introduce the local space $\mathcal{H}(K) \subset\left[H^{1}(K)\right]^{2}$ made of vector functions with constant divergence

$$
\mathcal{H}(K)=\left\{\mathbf{v} \in\left[H^{1}(K)\right]^{2}:|K| \operatorname{div} \mathbf{v}=\left(\int_{\partial K} \mathbf{v} \cdot \mathbf{n}_{e}^{K} \mathrm{~d} s\right) \text { in } K\right\},
$$

$\mathbf{n}_{e}^{K}$ being the outward unit normal to $K$. Then, we define the finite dimensional space $V_{h \mid K}$ as

$$
V_{h \mid K}=\left\{\mathbf{v} \in \mathcal{H}(K): \mathbf{v}=\underset{\mathbf{q} \in \mathcal{H}(K), \mathbf{q}=\mathbf{w} \text { on } \partial K}{\operatorname{argmin}}\|\nabla q\|_{2}, \mathbf{w} \in \mathbb{B}_{\partial K}\right\},
$$

where

$$
\mathbb{B}_{\partial K}=\left\{\mathbf{v} \in\left[C^{0}(\partial K)\right]^{2}: \mathbf{v}_{\mid e} \cdot \mathbf{t}_{e}^{K} \in \mathbb{P}_{1}(e), \mathbf{v}_{\mid e} \cdot \mathbf{n}_{e}^{K} \in \mathbb{P}_{2}(e) \forall e \in \mathcal{E}_{K}\right\},
$$

where $\mathbf{t}_{e}^{K}$ is the tangent vector defined as the counterclockwise rotation of $\mathbf{n}_{e}^{K}$ by $90^{\circ}$. Note that the space $V_{h \mid K}$ is well defined. Indeed, given a (piecewise polynomial) 


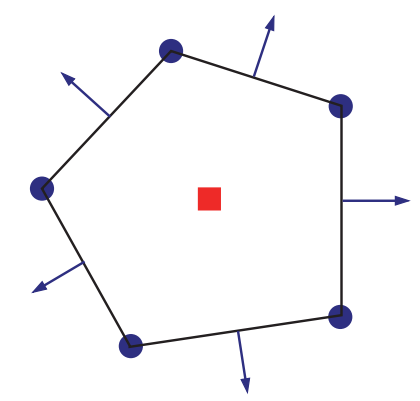

FIG. 3.1. Local degrees of freedom for velocities and pressures. The dots represent (vector) point values at vertices, while the arrows represent the point value of the normal component at the midpoint of the edge. The square represents the average.

boundary value $\left.\mathbf{v}\right|_{\partial K} \in\left[H^{1 / 2}(\partial K)\right]^{2}$, the associated function $\mathbf{v}$ inside the element $K$ is obtained by solving the following well posed Stokes-type problem:

$$
\begin{cases}\text { Find } \mathbf{v} \in\left[H^{1}(K)\right]^{2}, r \in L_{0}^{2}(K) & \text { such that } \\ -\Delta \mathbf{v}-\nabla r=\mathbf{0} & \text { in } K, \\ \operatorname{div} \mathbf{v}=c & \text { in } K, \\ \mathbf{v} \text { assigned on } \partial K, & \end{cases}
$$

where the equations are to be intended in the weak sense and the constant $c:=$ $\left(\int_{\partial K} \mathbf{v} \cdot \mathbf{n}_{K} \mathrm{~d} s\right) /|K|$ is compatible with the boundary conditions.

We remark that the space $V_{h \mid K}$ contains $H^{1}$ vector fields characterized by: (a) linear tangent component and quadratic normal component on each edge $e$; (b) constant divergence value on $K$; (c) minimum energy. Moreover, we note that, by standard regularity results for the Stokes problem on Lipschitz domain (see, e.g., [32]), the functions in $V_{h \mid K}$ turn out to be continuous in $\bar{K}$.

It is important to observe that, since the functions of $V_{h \mid K}$ are uniquely identified by their boundary values, the dimensions of $V_{h \mid K}$ and $\left(V_{h \mid K}\right)_{\mid \partial K}$ are equal, i.e., $\operatorname{dim}\left(V_{h \mid K}\right)=3 n, n$ being the number of edges of $K$. This leads to introducing the following $3 n$ degrees of freedom for the space $V_{h \mid K}$ (cf. the dots and arrows in Figure 3.1):

- the (vector) values of $\mathbf{v}$ at the vertices of $K$;

- the values of the normal components $\mathbf{v} \cdot \mathbf{n}_{e}^{K}$ at the midpoint of each edge of $K$.

Finally, it is immediate to verify that these degrees of freedom uniquely identify the restriction to $\partial K$ of the functions belonging to $V_{h \mid K}$.

The global velocity space $V_{h}$ is obtained by combining the local spaces $V_{h \mid K}$ accordingly to the local degrees of freedom, as is standard in finite elements $[20,15,13]$, and considering the homogeneous boundary conditions. We obtain the space

$$
V_{h}=\left\{\mathbf{v}_{h} \in C^{0}(\Omega):\left.\mathbf{v}_{h}\right|_{K} \in V_{h \mid K} \forall K \in \mathcal{T}_{h}, \mathbf{v}_{h}=\mathbf{0} \text { on } \partial \Omega\right\}
$$

with the degrees of freedom given by the (vector) values at all the internal vertices of $\mathcal{T}_{h}$ and the normal components $\mathbf{v} \cdot \mathbf{n}_{e}$ at the midpoints of all internal edges $e$ of the mesh.

The pressure space is simply given by the piecewise constant functions

$$
Q_{h}=\left\{q_{h} \in L_{0}^{2}(\Omega):\left.q_{h}\right|_{K} \in \mathbb{P}_{0}(K) \forall K \in \mathcal{T}_{h}\right\},
$$


and the degrees of freedom are one per element, given by the value of the function on the element (cf. the square in Figure 3.1).

3.2. Bilinear form and loading term. In this section we will discuss the construction of the local bilinear form appearing in (3.2).

The local bilinear forms $a_{h}^{K}(\cdot, \cdot): V_{h \mid K} \times V_{h \mid K} \rightarrow \mathbb{R}, K \in \mathcal{T}_{h}$, are assumed to be symmetric and satisfy the following consistency and stability assumptions.

(A1) Consistency: for all $h>0$ and for all $K \in \mathcal{T}_{h}$ it holds that

$$
a_{h}^{K}\left(\mathbf{p}, \mathbf{v}_{h}\right)=a^{K}\left(\mathbf{p}, \mathbf{v}_{h}\right) \quad \forall \mathbf{p} \in\left[\mathbb{P}_{1}(K)\right]^{2}, \mathbf{v}_{h} \in V_{h \mid K} .
$$

(A2) Stability: there exist two positive constants $\alpha_{*}$ and $\alpha^{*}$, independent of $h$ and of $K$, such that

$$
\alpha_{*} a^{K}\left(\mathbf{v}_{h}, \mathbf{v}_{h}\right) \leq a_{h}^{K}\left(\mathbf{v}_{h}, \mathbf{v}_{h}\right) \leq \alpha^{*} a^{K}\left(\mathbf{v}_{h}, \mathbf{v}_{h}\right) \quad \forall \mathbf{v}_{h} \in V_{h \mid K} .
$$

First of all, we observe that the local degrees of freedom allow us to compute exactly $a^{K}\left(\mathbf{p}, \mathbf{v}_{h}\right)$ for any $\mathbf{p} \in\left[\mathbb{P}_{1}(K)\right]^{2}$ and for any $\mathbf{v}_{h} \in V_{h \mid K}$. Indeed,

$$
\begin{aligned}
a^{K}\left(\mathbf{p}, \mathbf{v}_{h}\right) & =\nu \int_{K} \boldsymbol{\nabla}^{S} \mathbf{p}: \nabla^{S} \mathbf{v}_{h} \mathrm{~d} x \\
& =-\nu \int_{K} \operatorname{div}\left(\boldsymbol{\nabla}^{S} \mathbf{p}\right) \cdot \mathbf{v}_{h} \mathrm{~d} x+\nu \int_{\partial K}\left(\left(\boldsymbol{\nabla}^{S} \mathbf{p}\right) \mathbf{n}_{e}^{K}\right) \cdot \mathbf{v}_{h} \mathrm{~d} s,
\end{aligned}
$$

with $\mathbf{n}_{e}^{K}$ the outward unit normal to $K$. Therefore, since $\operatorname{div}\left(\boldsymbol{\nabla}^{S} \mathbf{p}\right)=\mathbf{0}$ and the functions $\mathbf{v}_{h} \in V_{h \mid K}$ are known explicitly on the boundary, the right-hand side of (3.5) can be computed exactly without knowing $\mathbf{v}_{h}$ in the interior of $K$.

We note that the practical implementation of the local stiffness matrices associated to the local bilinear forms $a_{h}(\cdot, \cdot)$ can be found, for the mimetic framework, in [8] or can be easily adapted by extending the VEM construction of [7] to the present case. Detailing such a construction is beyond the scope of the present paper. However, we remark that, in order for condition (3.6) to hold ( $h$-uniformly), some mesh regularity assumptions are needed. A possible choice, although not the more general one, is the following [7].

(A3) Mesh regularity: there exists a constant $\gamma>0$ such that for any $h>0$ every element $K \in \mathcal{T}_{h}$ is star-shaped with respect to a ball of radius $\geq \gamma h_{K}, h_{K}$ being the diameter of $K$. Moreover, we assume that there exists a constant $\gamma^{\prime}>0$ such that for any $h>0$ and for every $K \in \mathcal{T}_{h}$, the distance between any two vertices of $K$ is $\geq \gamma^{\prime} h_{K}$.

We remark that assumption (A3) is needed also to guarantee the validity of optimal interpolation error estimates [7].

We now discuss the construction of the loading term. For every $K \in \mathcal{T}_{h}$ and $\mathbf{v}_{h} \in V_{h \mid K}$ we set

$$
\overline{\mathbf{v}}_{K}:=\frac{1}{n} \sum_{i=1}^{n} \mathbf{v}_{h}\left(\mathbf{v}_{i}\right), \quad \mathbf{v}_{i}=\text { vertices of } K .
$$

We approximate $\mathbf{f}$ by a piecewise constant vector function $\mathbf{f}_{h}$ and we denote by $\mathbf{f}_{K}$ the restriction of $\mathbf{f}_{h}$ to $K$. We take as the value of $\mathbf{f}_{K}$ on each $K \in \mathcal{T}_{h}$ the average of f on $K$. This naturally leads to identifying $\mathbf{f}_{h}$ with an element of the dual space $V_{h}^{\prime}$ and to introducing the following duality pair:

$$
\left\langle\mathbf{f}_{h}, \mathbf{v}_{h}\right\rangle_{h}:=\sum_{K \in \mathcal{T}_{h}} \int_{K} \mathbf{f}_{K} \cdot \overline{\mathbf{v}}_{K} \mathrm{~d} x=\sum_{K \in \mathcal{T}_{h}}|K| \mathbf{f}_{K} \cdot \overline{\mathbf{v}}_{K} .
$$


3.3. Discrete problem. The results of the previous sections allow us to introduce the following VEM in velocity-pressure formulation for the approximation of the Stokes problem (2.1):

$$
\left\{\begin{array}{l}
\text { Find } \mathbf{u}_{h} \in V_{h}, p_{h} \in Q_{h} \text { such that } \\
a_{h}\left(\mathbf{u}_{h}, \mathbf{v}_{h}\right)+\left(\operatorname{div} \mathbf{v}_{h}, p_{h}\right)=\left\langle\mathbf{f}_{h}, \mathbf{v}_{h}\right\rangle_{h} \quad \forall \mathbf{v}_{h} \in V_{h}, \\
\left(\operatorname{div} \mathbf{u}_{h}, q_{h}\right)=0 \quad \forall q_{h} \in Q_{h} .
\end{array}\right.
$$

We preliminary remark that the divergence of any function $\mathbf{v}_{h}$ in $V_{h \mid K}$ is explicitly computable. Indeed, since div $\left.\mathbf{v}_{h}\right|_{K}$ is constant, we have

$$
\left.\operatorname{div} \mathbf{v}_{h}\right|_{K}=\frac{1}{|K|} \int_{K} \operatorname{div} \mathbf{v}_{h} \mathrm{~d} x=\frac{1}{|K|} \int_{\partial K} \mathbf{v}_{h} \cdot \mathbf{n}_{e}^{K} \mathrm{~d} s,
$$

where the right-hand side is computable as the functions in $V_{h \mid K}$ are explicitly known on the boundary. Hence, recalling that the functions in $Q_{h}$ are constant on each element, the divergence terms appearing in (3.10) are explicitly computable as there holds

$$
\left(\operatorname{div} \mathbf{v}_{h}, q_{h}\right)=\sum_{K \in \mathcal{T}_{h}} \int_{K} \operatorname{div} \mathbf{v}_{h} q_{h} \mathrm{~d} x=\sum_{K \in \mathcal{T}_{h}} q_{h \mid K} \int_{K} \operatorname{div} \mathbf{v}_{h} \mathrm{~d} x
$$

for all $\mathbf{v}_{h} \in V_{h}$ and $q_{h} \in Q_{h}$.

We remark that the VEM (3.10) is equivalent to the mimetic method for the Stokes problem introduced in $[8,10]$ (apart from the presence of the symmetric gradient instead of gradient, that constitutes a minor difference from the theoretical standpoint). In other words, under the present mesh conditions, it can be shown that the two families of methods are characterized by the same fundamental conditions of consistency and stability (although expressed in a different way). Therefore, stability and convergence results are easily derived from the results in [10] combined with the techniques of [7]. Therefore, we give only a brief summary of the main steps in the proof.

THEOREM 3.1. Let the assumptions (A1)-(A3) hold for the family of meshes $\left\{\mathcal{T}_{h}\right\}_{h>0}$. Then, the problems (3.10) are (uniformly) well posed for all $h>0$. Moreover, let $(\mathbf{u}, p)$ be the solution of (2.2), and let $\left(\mathbf{u}_{h}, p_{h}\right)$ be the solution of (3.10). Then it holds that

$$
\left\|\mathbf{u}-\mathbf{u}_{h}\right\|_{1}+\left\|p-p_{h}\right\|_{0} \leq C h\left(\sum_{K \in \mathcal{T}_{h}}\left(|\mathbf{u}|_{2, K}^{2}+|p|_{1, K}^{2}\right)\right)^{1 / 2},
$$

where the constant $C$ is independent of $h$.

Proof. We present a brief summary of the proof, that we divide into three steps.

Part 1. Following the same argument in [10], one can show that the discrete inf-sup condition holds:

$$
\sup _{\mathbf{v}_{h} \in V_{h} / \mathbf{0}} \frac{\left(\operatorname{div} \mathbf{v}_{h}, q_{h}\right)}{\left\|\mathbf{v}_{h}\right\|_{1}} \geq C_{\beta}\left\|q_{h}\right\|_{0} \quad \forall q_{h} \in Q_{h},
$$

with $C_{\beta}>0$ independent of $h$. Moreover, the coercivity condition (in this case on the whole $V_{h}$ )

$$
a_{h}\left(\mathbf{v}_{h}, \mathbf{v}_{h}\right) \geq C_{\alpha}\left\|\mathbf{v}_{h}\right\|_{1}^{2} \quad \forall \mathbf{v}_{h} \in V_{h},
$$

Copyright $@$ by SIAM. Unauthorized reproduction of this article is prohibited. 
with $C_{\alpha}>0$ independent of $h$, also holds due to the stability assumption (3.6) and the Korn inequality; see, for instance, [21]. Combining these two conditions (see [15]) the stability of the discrete problem in the norms $\|\cdot\|_{1}$ for $V_{h}$ and $\|\cdot\|_{0}$ for $Q_{h}$ follows.

Part 2. Let the bilinear form $\mathcal{B}_{h}:\left[V_{h} \times Q_{h}\right] \times\left[V_{h} \times Q_{h}\right] \rightarrow \mathbb{R}$ be defined by

$$
\mathcal{B}\left(\mathbf{v}_{h}, q_{h} ; \mathbf{w}_{h}, s_{h}\right)=a_{h}\left(\mathbf{v}_{h}, \mathbf{w}_{h}\right)+\left(\operatorname{div} \mathbf{w}_{h}, q_{h}\right)+\left(\operatorname{div} \mathbf{v}_{h}, s_{h}\right)
$$

for all $\mathbf{v}_{h}, \mathbf{w}_{h} \in V_{h}$ and $q_{h}, s_{h} \in Q_{h}$. Then the stability of the discrete problem implies the existence of an $h$-uniform constant $C_{s}>0$ such that, for all $\mathbf{u}_{I} \in V_{h}$ and $p_{I} \in Q_{h}$, there exists a couple $\left(\mathbf{v}_{h}, q_{h}\right) \in V_{h} \times Q_{h}$ such that

$$
\mathcal{B}_{h}\left(\mathbf{u}_{h}-\mathbf{u}_{I}, p_{h}-p_{I} ; \mathbf{v}_{h}, q_{h}\right) \geq C_{s}\left(\left\|\mathbf{u}_{h}-\mathbf{u}_{I}\right\|_{1}+\left\|p_{h}-p_{I}\right\|_{0}\right)
$$

and $\left\|\mathbf{v}_{h}\right\|_{1}+\left\|q_{h}\right\|_{0} \leq 1$. From the above stability bound (following steps that are very similar to the ones in the proof of Theorem $3.1 \mathrm{in} \mathrm{[7])} \mathrm{using} \mathrm{(A1)} \mathrm{and} \mathrm{after} \mathrm{some}$ calculation one obtains that for any $\mathcal{T}_{h}$-piecewise linear vector field $\mathbf{u}_{I I} \in\left[L^{2}(\Omega)\right]^{2}$ it holds that

$$
\begin{aligned}
\left\|\mathbf{u}_{h}-\mathbf{u}_{I}\right\|_{1}+\left\|p_{h}-p_{I}\right\|_{0} & \leq C_{s}^{-1} \mathcal{B}_{h}\left(\mathbf{u}_{h}-\mathbf{u}_{I}, p_{h}-p_{I} ; \mathbf{v}_{h}, q_{h}\right) \\
& =C_{s}^{-1}\left(T_{1}+T_{2}+T_{3}+T_{4}\right),
\end{aligned}
$$

where

$$
\begin{aligned}
& T_{1}:=\left\langle\mathbf{f}_{h}, \mathbf{v}_{h}\right\rangle_{h}-\left(\mathbf{f}, \mathbf{v}_{h}\right), \\
& T_{2}:=\mathcal{B}_{h}\left(\mathbf{u}-\mathbf{u}_{I}, p-p_{I} ; \mathbf{v}_{h}, q_{h}\right), \\
& T_{3}:=\sum_{K \in \mathcal{T}_{h}} a_{h}^{K}\left(\mathbf{u}_{I I}-\mathbf{u}, \mathbf{v}_{h}\right), \\
& T_{4}:=\sum_{K \in \mathcal{T}_{h}} a^{K}\left(\mathbf{u}-\mathbf{u}_{I I}, \mathbf{v}_{h}\right) .
\end{aligned}
$$

Part 3. A triangle inequality, property (A2), (3.14), and standard bounds yield

$$
\begin{aligned}
\left\|\mathbf{u}-\mathbf{u}_{h}\right\|_{1}+\left\|p-p_{h}\right\|_{0} & \leq C\left(\left|\left\langle\mathbf{f}_{h}, \mathbf{v}_{h}\right\rangle_{h}-\left(\mathbf{f}, \mathbf{v}_{h}\right)\right|+\left\|\mathbf{u}-\mathbf{u}_{I}\right\|_{1}\right. \\
& \left.+\left\|p-p_{I}\right\|_{0}+\left(\sum_{K \in \mathcal{T}_{h}}\left\|\mathbf{u}-\mathbf{u}_{I I}\right\|_{1, K}^{2}\right)^{1 / 2}\right),
\end{aligned}
$$

with the constant $C$ independent of $h$. The three last terms in the right-hand side of (3.15) are bounded by standard approximation estimates using assumption (A3); we give the details only for the first term. We recall definition (3.9) and that $\mathbf{f}_{h}$ is the piecewise constant vector function that is the average of $\mathbf{f}$ on each $K \in \mathcal{T}_{h}$. Moreover, we indicate with $\mathbf{v}_{K}$, for each $K \in \mathcal{T}_{h}$, the average of $\mathbf{v}_{h}$ on $K$. Simple algebra then yields

$$
\begin{aligned}
\left|\left\langle\mathbf{f}_{h}, \mathbf{v}_{h}\right\rangle_{h}-\left(\mathbf{f}, \mathbf{v}_{h}\right)\right| & \leq\left|\sum_{K \in \mathcal{T}_{h}} \int_{K} \mathbf{f}_{K} \cdot\left(\overline{\mathbf{v}}_{K}-\mathbf{v}_{K}\right) \mathrm{d} x\right| \\
& +\left|\sum_{K \in \mathcal{T}_{h}} \int_{K} \mathbf{f} \cdot\left(\mathbf{v}_{K}-\mathbf{v}_{h}\right) \mathrm{d} x\right|,
\end{aligned}
$$

where we used that $\int_{K} \mathbf{f} \cdot \mathbf{v}_{K}=\int_{K} \mathbf{f}_{K} \cdot \mathbf{v}_{K}$ for all $K \in \mathcal{T}_{h}$. The two terms on the right-hand side above are bounded using the Cauchy-Schwarz inequality and approximation estimates, recalling that $\left\|\mathbf{v}_{h}\right\|_{1} \leq 1$ (see part 2) and using $\left\|\mathbf{f}_{h}\right\|_{0, K} \leq$ $\|\mathbf{f}\|_{0, K} \leq C|\mathbf{u}|_{2, K}$ for all $K \in \mathcal{T}_{h}$. 
4. VEM for Stokes (II): Stream formulation. In this section we present our novel VEM in stream formulation for the approximation of the Stokes problem (2.1). The stream formulation hinges upon the introduction of a suitable stream function space (characterizing the divergence free subspace of discrete velocities) and it is equivalent to the velocity-pressure virtual element scheme (3.10).

Let us first introduce the following space of discrete divergence-free functions:

$$
\begin{aligned}
Z_{h} & =\left\{\mathbf{v}_{h} \in V_{h}: \int_{\Omega} \operatorname{div} \mathbf{v}_{h} q_{h} \mathrm{~d} x=0 \quad \forall q_{h} \in Q_{h}\right\} \\
& =\left\{\mathbf{v}_{h} \in V_{h}: \operatorname{div} \mathbf{v}_{h}=0\right\},
\end{aligned}
$$

where the equality above follows from the fact that $V_{h}$ has piecewise constant divergence. Moreover, we define the following (local) space of discrete stream functions:

$$
\left.\Phi_{h}\right|_{K}:=\left\{\phi \in H^{2}(K): \phi=\underset{\substack{\eta \in H^{2}(K) \\ \eta=v, \nabla \eta=\mathbf{v} \text { on } \partial K}}{\operatorname{argmin}}\|\nabla(\operatorname{curl} \eta)\|_{L^{2}(K)}^{2}, v \in \mathbb{B}_{\partial K}^{\prime}, \mathbf{v} \in \mathbb{B}_{\partial K}^{\prime \prime}\right\},
$$

where

$$
\begin{aligned}
& \mathbb{B}_{\partial K}^{\prime}=\left\{v \in C^{0}(\partial K): v_{\mid e} \in \mathbb{P}_{3}(e) \forall e \in \mathcal{E}_{K}\right\}, \\
& \mathbb{B}_{\partial K}^{\prime \prime}=\left\{\mathbf{v} \in\left[C^{0}(\partial K)\right]^{2}: \mathbf{v}_{\mid e} \cdot \mathbf{n}_{e}^{K} \in \mathbb{P}_{1}(e) \forall e \in \mathcal{E}_{K}\right\} .
\end{aligned}
$$

Note that the space $\left.\Phi_{h}\right|_{K}$ is well defined. Indeed, given the values on the boundary $\partial K$, the function $\phi$ inside the element $K$ is found by solving a fourth order elliptic problem

$$
\left\{\begin{array}{l}
-\Delta^{2} \phi=0 \quad \text { in } K, \\
\phi \text { assigned on } \partial K, \\
\nabla \phi \cdot \mathbf{n}_{e}^{K} \text { assigned on } \partial K,
\end{array}\right.
$$

and standard regularity results for fourth order elliptic problems on Lipschitz domain yield that the functions of $\left.\Phi_{h}\right|_{K}$ are in $C^{1}(K)$ (see, e.g., [32]).

It is easy to check that the following constitute a set of degrees of freedom for the space $\left.\Phi_{h}\right|_{K}$ (see Figure 4.1):

- the point values of $\phi$ at the vertices of $K$;

- the (vector) values of $\nabla \phi$ at the vertices of $K$.

The global stream function space $\Phi_{h}$ is obtained by combining the local spaces $\Phi_{h \mid K}$ accordingly to the local degrees of freedom, taking into account the boundary conditions and enforcing an additional constraint in order to neglect the global constant functions. We obtain the space

$$
\Phi_{h}=\left\{\phi_{h} \in C^{1}(\Omega):\left.\phi_{h}\right|_{K} \in \Phi_{h \mid K} \forall K \in \mathcal{T}_{h}, \operatorname{curl} \phi_{h}=\mathbf{0} \text { on } \partial \Omega, \int_{\partial \Omega} \phi_{h} \mathrm{~d} x=0\right\} .
$$

We preliminarily collect the following result.

Lemma 4.1. For every $\mathbf{v}_{h} \in Z_{h}$ there exists a unique $\phi_{h} \in \Phi_{h}$ such that

$$
\left.\left(\operatorname{curl} \phi_{h}\right)\right|_{\partial K}=\left.\mathbf{v}_{h}\right|_{\partial K} \quad \forall K \in \mathcal{T}_{h} .
$$




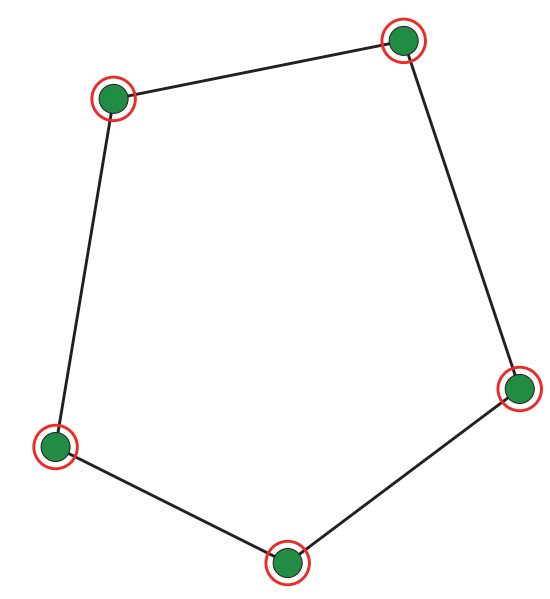

FIG. 4.1. Local degrees of freedom for the stream space $\Phi_{h}$. Dots represent point values and circles denote point values of the gradient.

Proof. Given $\mathbf{v}_{h} \in Z_{h}$, we will explicitly build $\phi_{h} \in \Phi_{h}$ such that (4.2) holds. Recalling the degrees of freedom of $\Phi_{h}$ (see Figure 4.1), we need to determine the values of $\phi_{h}$ and $\nabla \phi_{h}$ at all the mesh vertices. We preliminarily choose an arbitrary vertex $\tilde{\mathrm{v}}$ of the mesh and set $\phi_{h}(\tilde{\mathrm{v}})=C$, for some constant $C$ to be chosen later. Then, for any other vertex $\widehat{v}$ in the mesh, we build an oriented path $\gamma_{h}$ from $\tilde{v}$ to $\widehat{v}$ made of mesh edges and determine the value of $\phi_{h}$ at $\widehat{v}$ as follows:

$$
\phi_{h}(\widehat{\mathrm{v}}):=\phi_{h}(\tilde{\mathrm{v}})+\sum_{e \in \gamma_{h}} \int_{e} \mathbf{v}_{h} \cdot \mathbf{n}_{e}^{\gamma_{h}} \mathrm{~d} s
$$

where $\mathbf{n}_{e}^{\gamma_{h}}$ is the unit normal to edge $e$ that is obtained by a clockwise rotation of the unit tangent pointing in the direction of the oriented path. We note that the definition (4.3) is well posed since $\phi_{h}(\widehat{v})$ in (4.3) does not depend on the chosen path. Indeed, div $\mathbf{v}_{h}=0$ and the fact that the domain is simply connected imply that the sum in the right-hand side of (4.3) is zero on any closed path.

As a second step, using the definition of the operator curl, we define the value of $\nabla \phi_{h}$ at each vertex $\widehat{v}$ by setting

$$
\left(\operatorname{curl} \phi_{h}\right)(\widehat{\mathrm{v}}):=\mathbf{v}_{h}(\widehat{\mathrm{v}}) \quad \forall \widehat{\mathrm{v}} \in \mathcal{V}_{h} .
$$

Note that as $\mathbf{v}_{h} \in V_{h}$ we have $\left(\operatorname{curl} \phi_{h}\right)(\widehat{v})=\mathbf{0}$ for every $\widehat{\mathbf{v}} \in \mathcal{V}_{h}^{b}$. Finally, the initial constant $C$ is chosen in order to satisfy the zero integral condition in (4.1).

It is now easy to check that $\phi_{h}$ satisfies $\left.\left(\operatorname{curl} \phi_{h}\right)\right|_{e}=\left.\mathbf{v}_{h}\right|_{e}$ for all edges $e \in \mathcal{E}_{h}$. Indeed, let $e$ be an edge with extrema $\mathbf{v}_{1}$ and $\mathbf{v}_{2}$ (ordered in such a way that $\mathbf{t}_{e}$ points from $v_{1}$ to $v_{2}$ ); then (4.3) yields

$$
\int_{e}\left(\operatorname{curl} \phi_{h}\right) \cdot \mathbf{n}_{e} \mathrm{~d} s=\int_{e}\left(\nabla \phi_{h}\right) \cdot \mathbf{t}_{e} \mathrm{~d} s=\phi_{h}\left(\mathrm{v}_{2}\right)-\phi_{h}\left(\mathrm{v}_{1}\right)=\int_{e} \mathbf{v}_{h} \cdot \mathbf{n}_{e} \mathrm{~d} s .
$$

To conclude we first observe that $\mathbf{v}_{h} \cdot \mathbf{n}_{e}$ and $\operatorname{curl} \phi_{h} \cdot \mathbf{n}_{e}$ are polynomials of degree 2 on $e$ and they assume the same values at the two extrema $\mathrm{v}_{1}, \mathrm{v}_{2}$ (see (4.4)) and the same integral (see (4.5)); thus they coincide. Finally, $\mathbf{v}_{h} \cdot \mathbf{t}_{e}$ and $\operatorname{curl} \phi_{h} \cdot \mathbf{t}_{e}$ are equal on $e$ because they are linear functions taking the same values at the extrema (see (4.4)). This concludes the main assertion of the lemma. 
The uniqueness follows easily by the same lines and is thus shown briefly. Let $\left.\operatorname{curl} w_{h}\right|_{\partial K}=\mathbf{0}$ for all $K \in \mathcal{T}_{h}$ for some $w_{h} \in \Phi_{h}$, then the gradient values must vanish at all vertices in $\mathcal{V}_{h}$ and the same holds for all differences $w_{h}\left(v_{1}\right)-w_{h}\left(v_{2}\right)$ evaluated at the extrema $\mathrm{v}_{1}, \mathrm{v}_{2}$ of any edge $e \in \mathcal{E}_{h}$. The latter yields that $w_{h}$ assumes the same constant value; i.e., $w_{h}$ must be constant on $\Omega$. Finally, this implies that the function $w_{h}$ vanishes due to the zero average condition in the definition of $\Phi_{h}$.

Now, we are ready to prove the following characterization of the space $Z_{h}$.

Proposition 4.2. It holds that

$$
Z_{h}=\operatorname{curl} \Phi_{h}:=\left\{\operatorname{curl} \phi_{h}: \phi_{h} \in \Phi_{h}\right\} .
$$

Proof. We first introduce the space

$$
H^{\partial K}:=\left\{\overline{\mathbf{v}} \in\left[H^{1 / 2}(\partial K)\right]^{2}: \int_{\partial K} \overline{\mathbf{v}} \cdot \mathbf{n} d s=0\right\} .
$$

For every $\overline{\mathbf{v}} \in H^{\partial K}$ let us introduce the spaces $X_{1}^{K}(\overline{\mathbf{v}})$ and $X_{2}^{K}(\overline{\mathbf{v}})$ defined as follows:

$$
\begin{aligned}
& X_{1}^{K}(\overline{\mathbf{v}}):=\left\{\mathbf{v} \in\left[H^{1}(K)\right]^{2}: \operatorname{div} \mathbf{v}=0 \text { and }\left.\mathbf{v}\right|_{\partial K}=\overline{\mathbf{v}}\right\}, \\
& X_{2}^{K}(\overline{\mathbf{v}}):=\left\{\operatorname{curl} w: w \in H^{2}(K) \text { and }(\operatorname{curl} w)_{\partial K}=\overline{\mathbf{v}}\right\} .
\end{aligned}
$$

Using well-known results on the decomposition of 2D vector fields [27, Theorem 3.1], we deduce

$$
X_{1}^{K}(\overline{\mathbf{v}})=X_{2}^{K}(\overline{\mathbf{v}}) \quad \forall \overline{\mathbf{v}} \in H^{\partial K}
$$

Setting

$$
J_{K}(\mathbf{w}):=\|\nabla \mathbf{w}\|_{L^{2}(K)}^{2} \quad \forall \mathbf{w} \in\left[H^{1}(K)\right]^{2},
$$

and using (4.6) it is immediate to verify that for every $K \in \mathcal{T}_{h}$ and for every $\overline{\mathbf{v}} \in H^{\partial K}$ the following minimization problems admit unique solutions and there holds

$$
\underset{\mathbf{v} \in X_{1}^{K}(\overline{\mathbf{v}})}{\operatorname{argmin}} J_{K}(\mathbf{v})=\underset{\operatorname{curl} w \in X_{2}^{K}(\overline{\mathbf{v}})}{\operatorname{argmin}} J_{K}(\operatorname{curl} w) .
$$

We preliminarily observe that given

$$
\mathbf{v}_{h} \in Z_{h} \text { and the unique } w_{h} \in \Phi_{h}
$$

such that $\left.\left(\operatorname{curl} w_{h}\right)\right|_{\partial K}=\left.\mathbf{v}_{h}\right|_{\partial K}$ (see Lemma 4.1), it holds that

$$
\operatorname{curl} w_{h}=\mathbf{v}_{h} \text { in } K
$$

in view of $\left.\mathbf{v}_{h}\right|_{\partial K} \in H^{\partial K}$, equality (4.7), and the fact that (curl $\left.w_{h}\right)\left.\right|_{K}$ and $\left.\mathbf{v}_{h}\right|_{K}$ solve the minimization problem appearing on the right- and left-hand sides of (4.7), respectively. The latter is a consequence of $\left.\left.\mathbf{v}_{h}\right|_{K} \in X_{1}^{K}\left(\left.\mathbf{v}_{h}\right|_{\partial K}\right) \cap V_{h}\right|_{K}$ and $\left.w_{h}\right|_{K} \in$ $\left.X_{2}^{K}\left(\left.\mathbf{v}_{h}\right|_{\partial K}\right) \cap \Phi_{h}\right|_{K}$.

From the definition of the spaces $\left.\Phi_{h}\right|_{K}$ and $\left.V_{h}\right|_{K}$, it easily follows that for each $w_{h} \in \Phi_{h}$ there exists a unique $\mathbf{v}_{h} \in V_{h}$ such that $\left.\left(\operatorname{curl} w_{h}\right)\right|_{\partial K}=\left.\mathbf{v}_{h}\right|_{\partial K}$ for all $K \in \mathcal{T}_{h}$, and such $\mathbf{v}_{h}$ must satisfy $\operatorname{div} \mathbf{v}_{h}=0$. Therefore, due to (4.8), we immediately have that $\operatorname{curl} \Phi_{h} \subseteq Z_{h}$. On the other hand, Lemma 4.1 guarantees that for every $\mathbf{v}_{h}$ with 
$\operatorname{div} \mathbf{v}_{h}=0$ there exists a unique $w_{h} \in \Phi_{h}$ such that $\left.\left(\operatorname{curl} w_{h}\right)\right|_{\partial K}=\left.\mathbf{v}_{h}\right|_{\partial K}$ for all $K \in \mathcal{T}_{h}$. Hence, from (4.8) there follows $\operatorname{curl} w_{h}=\mathbf{v}_{h}$ in $\Omega$, i.e., $\operatorname{curl} \Phi_{h}=Z_{h}$.

In view of Proposition 4.2, the solution $\mathbf{u}_{h} \in V_{h}$ of (3.10) can be written as $\mathbf{u}_{h}=\operatorname{curl} \psi_{h}$, where $\psi_{h} \in \Phi_{h}$ solves

$$
\left\{\begin{array}{l}
\text { Find } \psi_{h} \in \Phi_{h} \text { such that } \\
a_{h}\left(\operatorname{curl} \psi_{h}, \operatorname{curl} \varphi_{h}\right)=\left\langle\mathbf{f}, \operatorname{curl} \varphi_{h}\right\rangle_{h} \quad \forall \varphi_{h} \in \Phi_{h} .
\end{array}\right.
$$

We will refer to (4.9) as to the virtual stream-formulation of problem (2.2).

Note that, as an immediate consequence of Lemma 4.1, the kernel of the curl operator on the space $\Phi_{h}$ is given by the trivial space $\{0\}$. Therefore, the strict positivity of $a_{h}(\cdot, \cdot)$ on $V_{h}$ immediately reflects on the strict positivity of $a_{h}(\operatorname{curl} \cdot$, curl $\cdot)$ on $\Phi_{h}$. This implies the invertibility of the discrete linear system in (4.9). The convergence of the solution $\mathbf{u}_{h}=\operatorname{curl} \psi_{h}$ to $\mathbf{u}$ follows immediately from Theorem 3.1 and the equivalence between the two formulations.

Remark 4.1. This novel virtual stream-formulation (4.9) exhibits several advantages from the computational point of view. First of all, the virtual stream formulation (4.9) results in a linear system of equations which is symmetric and positive definite (recall that the virtual element method (3.10) in velocity-pressure formulation lead to an indefinite linear system). This represents an advantage from the linear algebra point of view since a Cholesky factorization or a (preconditioned) conjugate gradient algorithm can be employed to solve the resulting linear system of equations. Moreover, formulation (4.9) has a much smaller number of unknowns compared to the velocitypressure formulation, and still keeping the same sparsity of the original method, as will be shown in section 5 .

4.1. Implementation issues. In this section, we present some details about the implementation of the method (4.9) and we observe that it does not hide any particular difficulty. The stream function construction presented in this paper covers all of the family of mimetic/VEM schemes in $[8,10]$ for the Stokes problem, as the stream function method can be implemented as a modification of such existing schemes. Indeed, one first needs to build the local (elementwise) stiffness matrices associated to the bilinear form $a_{h}^{K}(\cdot, \cdot), K \in \mathcal{T}_{h}$, and the local vectors representing the loading term $\left\langle\mathbf{f}_{h}, \cdot\right\rangle_{h}$ on $K$. This can be done identically to the mimetic method of $[8,10]$, or following the alternative way shown for the Laplace problem in [7]. The local stiffness matrices associated to the bilinear form $a_{h}^{K}(\operatorname{curl} \cdot, \operatorname{curl} \cdot)$ are then built by introducing local matrices $C U R L_{h}$ that represent the curl operator in terms of the degrees of freedom of $V_{h \mid K}$ and $\Phi_{h \mid K}$. For instance, given any $\varphi_{h} \in \Phi_{h}$, the vertex values of $\mathbf{v}_{h}=\operatorname{curl} \varphi_{h} \in V_{h}$ can be immediately computed using the values of $\nabla \varphi_{h}$ at the same vertices (which are, by definition, degrees of freedom of the space $\Phi_{h}$ ). In a similar way, we observe that the value of $\mathbf{v}_{h} \cdot \mathbf{n}_{e}$ at the edge midpoint $\mathrm{m}_{e}$ of $e$ can be computed as $\partial \varphi_{h} / \partial \mathbf{t}_{e}\left(\mathrm{~m}_{e}\right)$; recalling that $\varphi_{h}$ is a cubic function and using the Cavalieri-Simpson integration rule yields

$$
\frac{\partial \varphi_{h}}{\partial \mathbf{t}_{e}}\left(\mathrm{~m}_{e}\right)=\frac{3}{2|e|}\left(\varphi_{h}\left(\mathrm{v}^{\prime}\right)-\varphi_{h}(\mathrm{v})\right)-\frac{1}{4}\left(\frac{\partial \varphi_{h}}{\partial \mathbf{t}_{e}}\left(\mathrm{v}^{\prime}\right)+\frac{\partial \varphi_{h}}{\partial \mathbf{t}_{e}}(\mathrm{v})\right),
$$

$\mathrm{v}, \mathrm{v}^{\prime}$ being the two (ordered) vertices that are extrema for edge $e$. Note that the righthand side of the above relation is expressed in terms of the degrees of freedom of $\Phi_{h}$; i.e., of the vertex values of $\varphi_{h}$ and $\nabla \varphi_{h}$.

Similar arguments apply to the construction of the loading vector. 
The boundary conditions in our virtual stream-formulation have been imposed on the stream function $\psi_{h}$ in the following way: We set to zero the nodal values of $\nabla \psi_{h}$, and we impose $\psi_{h}=0$ on the whole boundary in order to fix the constant value of the stream function on the boundary. We note that there is no impact on the solution with the particular choice employed to fix that constant.

5. Numerical tests. In this section we test our virtual stream method (4.9) and compare its numerical performance with the one of the classical scheme (3.10). Both schemes have been implemented using MATLAB. The resulting linear systems have been solved using mldivide function, which employs direct methods.

In what follows, we consider two different benchmark problems defined on the computational domain $\Omega:=(0,1)^{2}$ and we employ the following types of mesh (see also Figures 5.1-5.2):

- $\mathcal{T}_{h}^{1}$ : Triangular mesh.

- $\mathcal{T}_{h}^{2}$ : Structured hexagonal meshes.

- $\mathcal{T}_{h}^{3}$ : Nonstructured hexagonal meshes made of convex hexagons.

- $\mathcal{T}_{h}^{4}$ : Regular subdivisions of the domain in $N \times N$ subsquares.

- $\mathcal{T}_{h}^{5}$ : Trapezoidal meshes which consist of partitions of the domain into $N \times N$ congruent trapezoids, all similar to the trapezoid with vertices $(0,0),\left(\frac{1}{2}, 0\right)$, $\left(\frac{1}{2}, \frac{2}{3}\right)$, and $\left(0, \frac{1}{3}\right)$.

- $\mathcal{T}_{h}^{6}$ : Meshes built from $\mathcal{T}_{h}^{1}$ considering the middle point of each edge as a new vertex, that is then moved randomly; note that these meshes contain nonconvex elements.
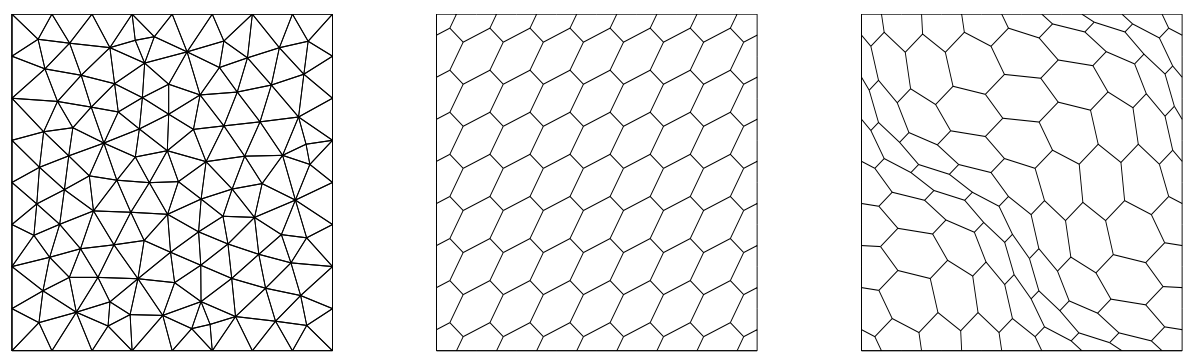

FIG. 5.1. Sample meshes: $\mathcal{T}_{h}^{1}$ (left), $\mathcal{T}_{h}^{2}$ (middle), and $\mathcal{T}_{h}^{3}$ (right) for $h=1 / 8$.
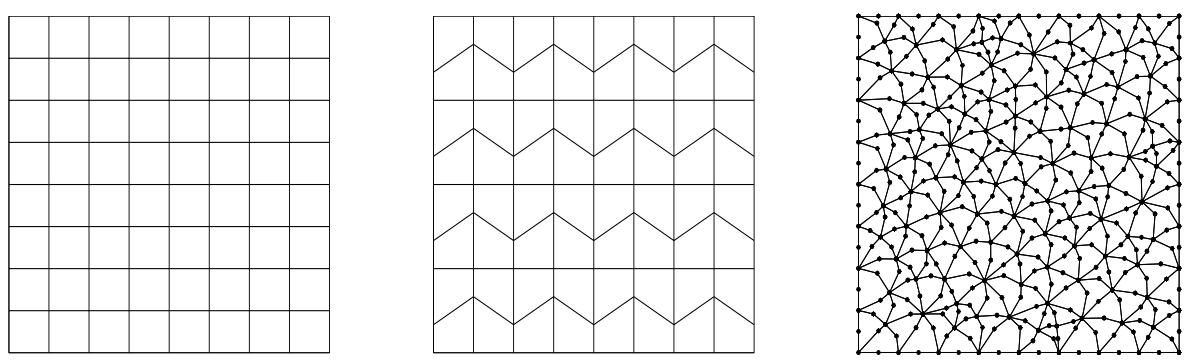

FIG. 5.2. Sample meshes: $\mathcal{T}_{h}^{4}$ (left), $\mathcal{T}_{h}^{5}$ (middle), and $\mathcal{T}_{h}^{6}$ (right) for $h=1 / 8$. 
To test the convergence properties of the methods, we introduce the following discrete maximum norm: for any sufficiently regular function $\mathbf{v}$,

$$
\left.|||\mathbf{v}|\right|_{0, \infty}:=\max _{\mathbf{v} \in \mathcal{V}_{h}}|\mathbf{v}(\mathbf{v})|_{\infty}
$$

where $\mathcal{V}_{h}$ represents the set of vertices of $\mathcal{T}_{h}$ and $|\cdot|_{\infty}$ denotes the $l^{\infty}$ vector norm. We also introduce the following discrete $H^{1}$-like norm:

$$
\|\mathbf{v}\|_{1,2}:=\left(\sum_{e \in \mathcal{E}_{h}} h_{e}\left\|\frac{\partial \mathbf{v}}{\partial \mathbf{t}_{e}}\right\|_{0, e}^{2}\right)^{1 / 2}
$$

Accordingly, we denote by

$$
E_{0, \infty}^{h}:=|| \mathbf{u}-\mathbf{u}_{h}\left|\left\|_{\infty}, \quad E_{1,2}^{h}:=\left.\left|\| \mathbf{u}-\mathbf{u}_{h}\right|\right|_{1,2}\right.\right.
$$

the corresponding errors, and we measure the experimental order of convergence as

$$
R:=\frac{\log \left(E / E^{\prime}\right)}{\log \left(h / h^{\prime}\right)},
$$

where $h$ and $h^{\prime}$ denote two consecutive meshsizes and $E$ and $E^{\prime}$ denote the associated errors.

5.1. Stokes problem with analytical solution. The first benchmark problem is taken from [4]. Choosing $\nu=1$ and the load $\mathbf{f}$ as

$$
\mathbf{f}(x, y)=\left[\begin{array}{c}
-4 \pi^{2} \cos (2 \pi x) \sin (2 \pi y)+2 \pi^{2} \sin (2 \pi y)-y^{2} \\
4 \pi^{2} \cos (2 \pi y) \sin (2 \pi x)-2 \pi^{2} \sin (2 \pi x)-2 x y
\end{array}\right]
$$

the solution $(\mathbf{u}, p)$ of problem (2.2) is given by

$$
\begin{gathered}
u_{1}(x, y)=-\cos (2 \pi x) \sin (2 \pi y)+\sin (2 \pi y), \\
u_{2}(x, y)=\sin (2 \pi x) \cos (2 \pi y)-\sin (2 \pi x), \\
p(x, y)=x y^{2}-\frac{1}{6} .
\end{gathered}
$$

In what follows, we report the numerical results obtained using the virtual velocity pressure method (3.10), and the virtual stream formulations (4.9). In the virtual stream formulation, the following boundary conditions have been employed for the stream function $\psi_{h}: \nabla \psi_{h}=(0,0)$ and $\psi_{h}=0$ on the whole boundary.

Table 5.1 shows the convergence history of the virtual velocity pressure method (3.10) applied to our test problem with five different family of meshes, while Table 5.2 collects the corresponding results when the virtual stream formulation (4.9) is applied. The tables include the number of degrees of freedom (dofs), the number nnz of nonzero matrix elements, the convergence rates $R$, the total time TT(s) in seconds used for computing the approximate solutions, and the discrete errors $E_{0, \infty}^{h}$ and $E_{1,2}^{h}$.

We note that the results reported in the tables confirm, for both methods, the first order convergence rate in the discrete $H^{1}$ like norm (in agreement with Theorem 3.1) and show a quadratic rate in the discrete $L^{\infty}$ norm. This holds for all the considered meshes. Moreover, since the two methods are equivalent, it is not surprising to note 
TABLE 5.1

Approximation of the velocity $\mathbf{u}$ : Convergence analysis of the virtual velocity-pressure method (3.10).

\begin{tabular}{|c|c|c|c|c|c|c|c|c|}
\hline Mesh & $1 / h$ & dof & $\mathrm{nnz}$ & $E_{0, \infty}^{h}$ & $R_{0, \infty}$ & $E_{1,2}^{h}$ & $R_{1,2}$ & $\mathrm{TT}(\mathrm{s})$ \\
\hline \multirow{5}{*}{$\mathcal{T}_{h}^{1}$} & 8 & 697 & 11558 & $8.7465 \mathrm{e}-2$ & - & $2.3821 \mathrm{e}-0$ & $\overline{-}$ & 1.36 \\
\hline & 16 & 2952 & 52321 & $3.4740 \mathrm{e}-2$ & 1.33 & $1.2100 \mathrm{e}-0$ & 0.98 & 3.86 \\
\hline & 32 & 12012 & 218947 & 7.7637e-3 & 2.16 & $6.2742 \mathrm{e}-1$ & 0.95 & 18.81 \\
\hline & 64 & 48899 & 903116 & $2.1290 \mathrm{e}-3$ & 1.87 & $3.1602 \mathrm{e}-1$ & 0.99 & 170.92 \\
\hline & 128 & 198496 & 3690320 & $5.6025 \mathrm{e}-4$ & 1.93 & $1.5800 \mathrm{e}-1$ & 1.00 & 2075.83 \\
\hline \multirow{5}{*}{$\mathcal{T}_{h}^{2}$} & 8 & 146 & 3781 & $7.6563 \mathrm{e}-1$ & - & $3.7968 \mathrm{e}-0$ & - & 0.34 \\
\hline & 16 & 546 & 16620 & $3.9845 \mathrm{e}-1$ & 0.94 & $2.1247 \mathrm{e}-0$ & 0.84 & 0.59 \\
\hline & 32 & 2114 & 68427 & $1.4242 \mathrm{e}-1$ & 1.48 & $9.2731 \mathrm{e}-1$ & 1.20 & 1.56 \\
\hline & 64 & 8322 & 279846 & $4.0863 \mathrm{e}-2$ & 1.80 & $3.7620 \mathrm{e}-1$ & 1.30 & 6.53 \\
\hline & 128 & 33026 & 1154059 & $1.0650 \mathrm{e}-2$ & 1.94 & $1.6652 \mathrm{e}-1$ & 1.18 & 93.90 \\
\hline \multirow{5}{*}{$\mathcal{T}_{h}^{3}$} & 8 & 146 & 3851 & $9.2800 \mathrm{e}-1$ & - & $3.9456 \mathrm{e}-0$ & - & 0.34 \\
\hline & 16 & 546 & 17117 & $5.8806 \mathrm{e}-1$ & 0.66 & $2.5752 \mathrm{e}-0$ & 0.62 & 0.63 \\
\hline & 32 & 2114 & 71887 & $2.6751 \mathrm{e}-1$ & 1.14 & $1.2899 \mathrm{e}-0$ & 1.00 & 1.60 \\
\hline & 64 & 8322 & 294709 & $9.2930 \mathrm{e}-2$ & 1.53 & $5.1945 \mathrm{e}-1$ & 1.31 & 6.78 \\
\hline & 128 & 33026 & 1193617 & $2.6568 \mathrm{e}-2$ & 1.81 & $2.0835 \mathrm{e}-1$ & 1.32 & 57.7 \\
\hline \multirow{5}{*}{$\mathcal{T}_{h}^{4}$} & 8 & 275 & 3960 & $5.4032 \mathrm{e}-1$ & - & $3.0340 \mathrm{e}-0$ & - & 0.43 \\
\hline & 16 & 1187 & 19410 & $1.8201 \mathrm{e}-1$ & 1.57 & $1.2733 \mathrm{e}-0$ & 1.25 & 1.00 \\
\hline & 32 & 4931 & 85284 & $5.0021 \mathrm{e}-2$ & 1.86 & $5.4736 \mathrm{e}-1$ & 1.22 & 3.71 \\
\hline & 64 & 20099 & 356798 & $1.2863 \mathrm{e}-2$ & 1.96 & $2.5782 \mathrm{e}-1$ & 1.09 & 22.6 \\
\hline & 128 & 81154 & 1433296 & $3.2361 \mathrm{e}-3$ & 1.99 & $1.2669 \mathrm{e}-1$ & 1.03 & 170.31 \\
\hline \multirow{5}{*}{$\mathcal{T}_{h}^{6}$} & 8 & 339 & 9428 & $4.8894 \mathrm{e}-1$ & - & $3.0986 \mathrm{e}-0$ & - & 0.88 \\
\hline & 16 & 1603 & 49412 & $1.5073 \mathrm{e}-1$ & 1.70 & $1.6231 \mathrm{e}-0$ & 0.93 & 1.99 \\
\hline & 32 & 6771 & 218348 & $4.2964 \mathrm{e}-2$ & 1.81 & $8.3954 \mathrm{e}-1$ & 0.95 & 7.46 \\
\hline & 64 & 27507 & 904488 & $1.0800 \mathrm{e}-2$ & 1.99 & $4.3331 \mathrm{e}-1$ & 0.95 & 54.50 \\
\hline & 128 & 111875 & 3713168 & $2.3505 \mathrm{e}-3$ & 2.20 & $2.1768 \mathrm{e}-1$ & 0.99 & 684.78 \\
\hline
\end{tabular}

that the error values reported in Table 5.1 and the corresponding ones of Table 5.2 are almost identical. The negligible discrepancy is related to the numerical round-off associated to the different sequence of computations performed by the two methods.

Finally, with the aim of performing a comparison between our novel virtual stream method and the virtual velocity-pressure method, we remark (see third column in Tables 5.1 and 5.2) that the number of dofs employed by the virtual stream method (4.9) is much smaller than the one used by the original scheme (3.10). For instance, in the triangular case the reduction factor is greater than two. Note that the reduction of the dofs also has important consequences on the number of nonzero elements in the matrices (see the fourth column in Tables 5.1 and 5.2). Finally, although the comparison may be code depending, we report (last column in the tables) the total time needed by the two algorithms to assemble and solve the linear systems. Again the advantage of the virtual stream formulation is clear.

5.2. The lid-driven cavity problem. The second benchmark example is the so called lid-driven cavity problem which is a standard test problem, for which there is no exact solution, typically employed to validate numerical methods for fluids (see, for instance, $[26,37])$. The $2 \mathrm{D}$ lid-driven cavity problem describes the flow in a rectangular container which is driven by the uniform motion of one lid.

The problem is set up with the following boundary condition: $\mathbf{u}=(1,0)$ on the top lid and $\mathbf{u}=(0,0)$ elsewhere, while the source function $\mathbf{f}$ is set equal to $\mathbf{0}$ and the viscosity $\nu$ equal to 1 .

Due to the change of boundary conditions, two singularities appear at the top corners of the domain. 
TABLE 5.2

Approximation of the velocity $\mathbf{u}$ : Convergence analysis of the virtual stream method (4.9).

\begin{tabular}{|c|c|c|c|c|c|c|c|c|}
\hline Mesh & $1 / h$ & dof & nnz & $E_{0, \infty}^{h}$ & $R_{0, \infty}$ & $E_{1,2}^{h}$ & $R_{1,2}$ & $\mathrm{TT}(\mathrm{s})$ \\
\hline \multirow{5}{*}{$\mathcal{T}_{h}^{1}$} & 8 & 273 & 5031 & $8.7466 \mathrm{e}-2$ & - & $2.3822 \mathrm{e}-0$ & - & 1.19 \\
\hline & 16 & 1212 & 24102 & $3.4752 \mathrm{e}-2$ & 1.33 & $1.2100 \mathrm{e}-0$ & 0.98 & 3.38 \\
\hline & 32 & 5040 & 103140 & $7.7743 \mathrm{e}-3$ & 2.16 & $6.2742 \mathrm{e}-1$ & 0.95 & 14.14 \\
\hline & 64 & 20739 & 429867 & $2.1300 \mathrm{e}-3$ & 1.87 & $3.1602 \mathrm{e}-1$ & 0.99 & 99.92 \\
\hline & 128 & 84633 & 1765593 & $5.6141 \mathrm{e}-4$ & 1.92 & $1.5800 \mathrm{e}-1$ & 1.00 & 1461.64 \\
\hline \multirow{5}{*}{$\mathcal{T}_{h}^{2}$} & 8 & 96 & 2672 & $7.6296 \mathrm{e}-1$ & - & $3.7972 \mathrm{e}-0$ & - & 0.30 \\
\hline & 16 & 384 & 12710 & $3.9766 \mathrm{e}-1$ & 0.94 & $2.1249 \mathrm{e}-0$ & 0.84 & 0.55 \\
\hline & 32 & 1536 & 55158 & $1.4217 \mathrm{e}-1$ & 1.48 & $9.2737 \mathrm{e}-1$ & 1.20 & 1.38 \\
\hline & 64 & 6144 & 229518 & $4.0791 \mathrm{e}-2$ & 1.80 & $3.7621 \mathrm{e}-1$ & 1.30 & 5.43 \\
\hline & 128 & 24576 & 939300 & $1.0632 \mathrm{e}-2$ & 1.94 & $1.6652 \mathrm{e}-1$ & 1.17 & 33.65 \\
\hline \multirow{5}{*}{$\mathcal{T}_{h}^{3}$} & $\overline{8}$ & 96 & 2676 & $9.2770 \mathrm{e}-1$ & - & $3.9448 \mathrm{e}-0$ & - & 0.25 \\
\hline & 16 & 384 & 12762 & $5.8785 \mathrm{e}-1$ & 0.66 & $2.5750 \mathrm{e}-0$ & 0.62 & 0.42 \\
\hline & 32 & 1536 & 55386 & $2.6743 \mathrm{e}-1$ & 1.14 & $1.2899 \mathrm{e}-0$ & 1.00 & 1.15 \\
\hline & 64 & 6144 & 230490 & $9.2912 \mathrm{e}-2$ & 1.53 & $5.1943 \mathrm{e}-1$ & 1.31 & 4.16 \\
\hline & 128 & 24576 & 940122 & $2.6563 \mathrm{e}-2$ & 1.81 & $2.0835 \mathrm{e}-1$ & 1.32 & 27.03 \\
\hline \multirow{5}{*}{$\mathcal{T}_{h}^{4}$} & 8 & 147 & 2065 & $5.3945 \mathrm{e}-1$ & - & $3.0340 \mathrm{e}-0$ & - & 0.45 \\
\hline & 16 & 675 & 11123 & $1.8179 \mathrm{e}-1$ & 1.57 & $1.2733 \mathrm{e}-0$ & 1.25 & 0.88 \\
\hline & 32 & 2883 & 50705 & $4.9967 \mathrm{e}-2$ & 1.86 & $5.4736 \mathrm{e}-1$ & 1.22 & 3.03 \\
\hline & 64 & 11907 & 215651 & $1.2849 \mathrm{e}-2$ & 1.96 & $2.5782 \mathrm{e}-1$ & 1.09 & 14.09 \\
\hline & 128 & 48387 & 897833 & $3.2327 \mathrm{e}-3$ & 1.99 & $1.2669 \mathrm{e}-1$ & 1.03 & 130.76 \\
\hline \multirow{5}{*}{$\mathcal{T}_{h}^{6}$} & 8 & 243 & 6705 & $4.8976 \mathrm{e}-1$ & - & $3.0986 \mathrm{e}-0$ & - & 1.05 \\
\hline & 16 & 1179 & 36765 & $1.5089 \mathrm{e}-1$ & 1.70 & $1.6231 \mathrm{e}-0$ & 0.93 & 1.85 \\
\hline & 32 & 5031 & 165843 & $4.2963 \mathrm{e}-2$ & 1.81 & $8.3953 \mathrm{e}-1$ & 0.95 & 6.74 \\
\hline & 64 & 20535 & 692937 & $1.0816 \mathrm{e}-2$ & 1.99 & $4.3331 \mathrm{e}-1$ & 0.96 & 42.22 \\
\hline & 128 & 83715 & 2856303 & $2.3514 \mathrm{e}-3$ & 2.20 & $2.1768 \mathrm{e}-1$ & 0.99 & 495.53 \\
\hline
\end{tabular}

In what follows, we report the numerical results obtained using the virtual stream formulation (4.9), where the following boundary conditions for the stream function $\psi_{h}$ have been employed: $\nabla \psi_{h}=(0,1)$ on the top lid and $\nabla \psi_{h}=(0,0)$ elsewhere; $\psi_{h}=0$ on the whole boundary.

We first employed a triangular mesh (family $\mathcal{T}_{h}^{1}$ ) with $h=1 / 64$ : Figure 5.3 reports the first and second velocity component profile, while Figure 5.4 shows the velocity field and the stream function.
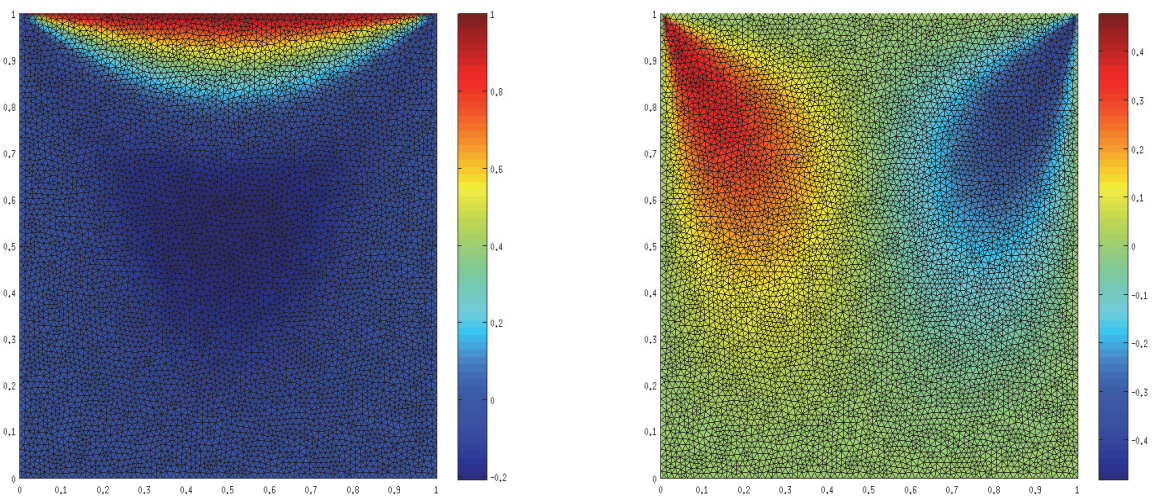

FIG. 5.3. The first and second velocity component profiles for the lid-driven cavity problem obtained with a triangular mesh (family $\mathcal{T}_{h}^{1}, h=1 / 64$ ). 

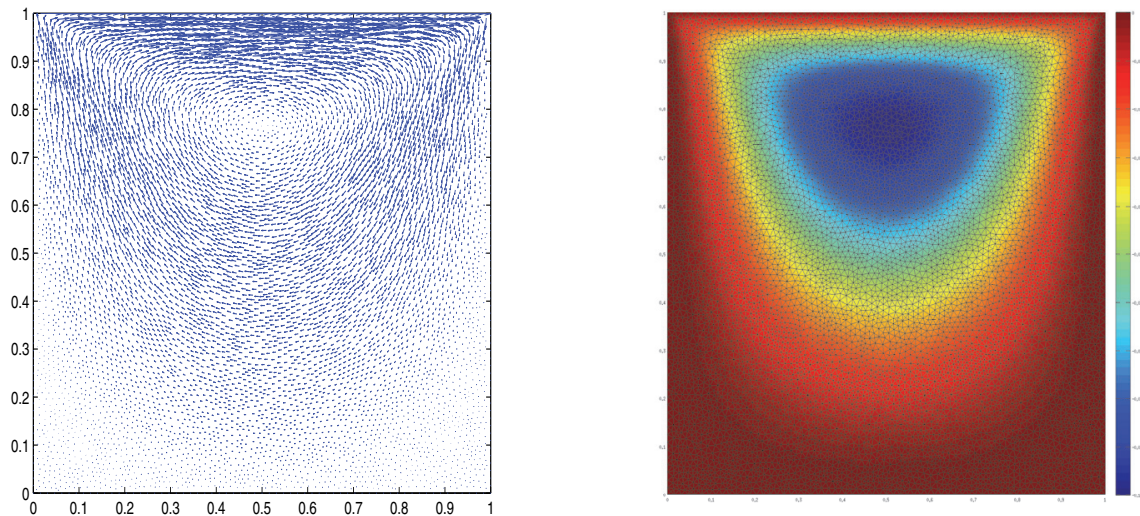

FIG. 5.4. The velocity profile and the stream function for the lid-driven cavity problem obtained with a triangular mesh (family $\mathcal{T}_{h}^{1}, h=1 / 64$ ).
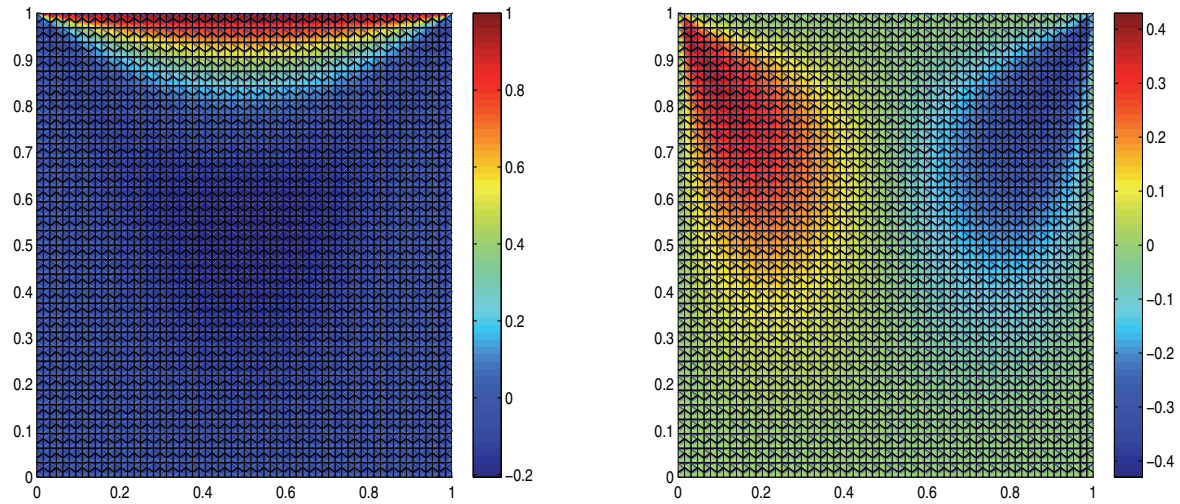

FIG. 5.5. The first and second velocity component profiles for the lid-driven cavity problem obtained with a trapezoidal mesh (family $\mathcal{T}_{h}^{5}, h=1 / 64$ ).
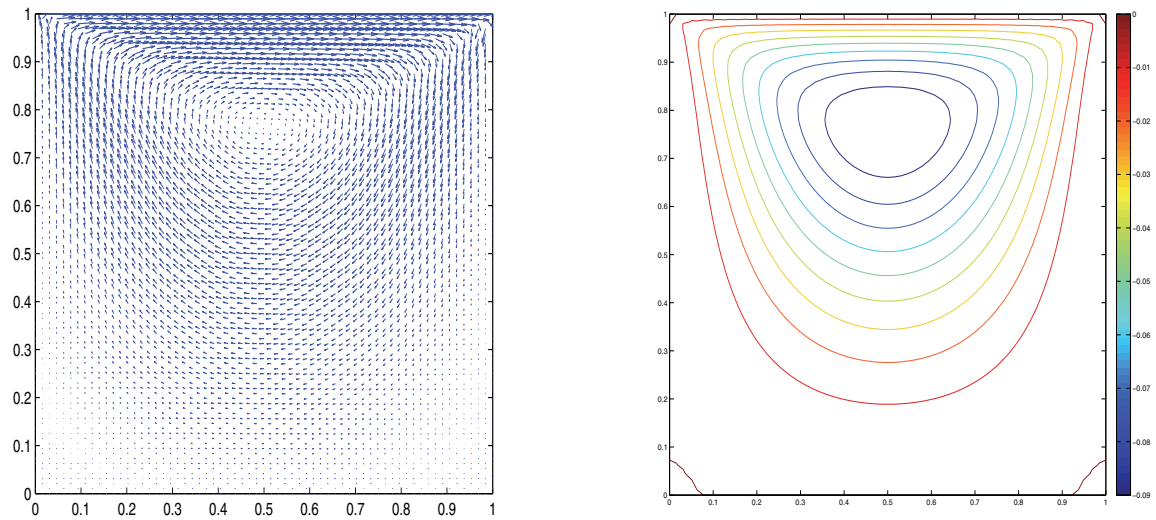

FIG. 5.6. The velocity profile and the streamlines for the lid-driven cavity problem obtained with a trapezoidal mesh (family $\mathcal{T}_{h}^{5}, h=1 / 64$ ). 
The second set of experiments have been run on trapezoidal mesh (family $\mathcal{T}_{h}^{5}$ ) with $h=1 / 64$. Figure 5.5 shows the first and second velocity component profile, and Figure 5.6 shows the velocity field and the streamlines.

The results obtained are in full agreement with those of $[26,37]$ and show both the stability and accuracy of our new virtual stream method also in the presence of jumping boundary conditions.

6. Conclusions. We have proposed and analyzed a new VEM for the approximation of the Stokes problem. The new formulation relies on construction of a suitable space of discrete divergence-free functions for the approximate velocities. We have showed that our virtual stream-formulation is equivalent to a virtual element velocity-pressure scheme of the Stokes problem. Exploiting such an equivalence, we have proved that the proposed scheme is well posed and the discrete solution converges to the continuous ones with optimal rate. We have also showed that our virtual stream-formulation leads to a linear system of equations that is much smaller compared to standard velocity-pressure schemes for Stokes problem and it is also symmetric and positive definite, and therefore it is very attractive from the computational point of view. Our theoretical results have been validated throughout a wide series of numerical experiments.

\section{REFERENCES}

[1] P. F. Antonietti, L. Beirão da Veiga, C. Lovadina, and M. Verani, Hierarchical a posteriori error estimators for the mimetic discretization of elliptic problems, SIAM J. Numer. Anal., 51 (2013), pp. 654-675.

[2] P. F. Antonietti, L. Beirão da Veiga, and M. Verani, A mimetic discretization of elliptic obstacle problems, Math. Comp., 82 (2013), pp. 1379-1400.

[3] P. F. Antonietti, N. Bigoni, and M. Verani, Mimetic discretizations of elliptic control problems, J. Sci. Comput., 56 (2013), pp. 14-27.

[4] F. Auricchio, L. Beirão da Veiga, C. Lovadina, and A. Reali, An analysis of some mixedenhanced finite element for plane linear elasticity, Comput. Methods Appl. Mech. Engrg., 194 (2005), pp. 2947-2968.

[5] L. Beirấ DA VeIga, A residual based error estimator for the mimetic finite difference method, Numer. Math., 108 (2008), pp. 387-406.

[6] L. Beirão da Veiga, F. Brezzi, and L. D. Marini, Virtual elements for linear elasticity problems, SIAM J. Numer. Anal., 51 (2013), pp. 794-812.

[7] L. Beirão da Veiga, F. Brezzi, A. Cangiani, G. Manzini, L. D. Marini, and A. Russo, Basic principles of virtual element methods, Math. Models Methods Appl. Sci., 23 (2013), pp. 199-214.

[8] L. Beirão da Veiga, V. Gyrya, K. Lipnikov, and G. Manzini, Mimetic finite difference method for the Stokes problem on polygonal meshes, J. Comput. Phys., 228 (2009), pp. 7215-7232.

[9] L. Beirão da Veiga and K. Lipnikov, A mimetic discretization of the Stokes problem with selected edge bubbles, SIAM J. Sci. Comput., 32 (2010), pp. 875-893.

[10] L. BeirÃo da Veiga, K. Lipnikov, and G. Manzini, Error analysis for a mimetic discretization of the steady Stokes problem on polyhedral meshes, SIAM J. Numer. Anal., 48 (2010), pp. 1419-1443.

[11] L. Beirão DA Veiga And G. Manzini, A virtual element method with arbitrary regularity, IMA J. Numer. Anal., to appear.

[12] L. Beirão da Veiga and D. Mora, A mimetic discretization of the Reissner-Mindlin plate bending problem, Numer. Math., 117 (2011), pp. 425-462.

[13] S. C. Brenner and R. L. Scott, The Mathematical Theory of Finite Element Methods, Texts Appl. Math. 15, 3rd ed., Springer-Verlag, New York, 2008.

[14] F. Brezzi, A. Buffa, And K. Lipnikov, Mimetic finite differences for elliptic problems, M2AN Math. Model. Numer. Anal., 43 (2009), pp. 277-295.

[15] F. Brezzi And M. Fortin, Mixed and Hybrid Finite Element Methods, Springer-Verlag, New York, 1991. 
[16] F. Brezzi, K. Lipnikov, and M. Shashkov, Convergence of mimetic finite difference method for diffusion problems on polyhedral meshes, SIAM J. Numer. Anal., 43 (2005), pp. 18721896.

[17] F. Brezzi, K. Lipnikov, And V. Simoncini, A family of mimetic finite difference methods on polygonal and polyhedral meshes, Math. Models Methods Appl. Sci., 15 (2005), pp. 15331551.

[18] F. Brezzi and L. D. Marini, Virtual elements for plate bending problems, Comput. Methods Appl. Mech. Engrg., 253 (2013), pp. 455-462.

[19] Z. Cai, C. Tong, P. S. Vassilevski, And C. WAng, Mixed finite element methods for incompressible flow: Stationary Stokes equations, Numer. Methods Partial Differential Equations, 26 (2010), pp. 957-978.

[20] P. G. Ciarlet, The Finite Element Method for Elliptic Problems, North-Holland, Amsterdam, 1978.

[21] P. G. Ciarlet, Mathematical Elasticity Vol. 1. Three Dimensional Elasticity, North-Holland, Amsterdam, 1987.

[22] M. Crouzeix And P.-A. Raviart, Conforming and nonconforming finite element methods for solving the stationary Stokes equations, RAIRO Serie Rouge, 7 (1973), pp. 33-75.

[23] J. Droniou and R. Eymard, Study of the mixed finite volume method for Stokes and NavierStokes equations, Numer. Methods Partial Differential Equations, 25 (2009), pp. 137-171.

[24] J. Droniou, R. Eymard, T. GallouËt, and R. Herbin, A unifed approach to mimetic finite difference, hybrid finite volume and mixed finite volume methods, Math. Models Methods Appl. Sci., 20 (2010), pp. 265-295.

[25] R. Eymard, T. Gallouët, R. Herbin, and J.-C. Latche, Convergence of the MAC scheme for the compressible Stokes equations, SIAM J. Numer. Anal., 48 (2010), pp. 2218-2246.

[26] L. P. Franca, S. L. Frey, and T. J. R. Hughes, Stabilized finite element methods: I. Application to the advective-diffusive model, Comput. Methods Appl. Mech. Engrg., 95 (1992), pp. 253-276.

[27] V. Girault And P. A. Raviart, Finite Element Methods for Navier-Stokes Equations, Springer-Verlag, Berlin, 1986.

[28] S. KRELL, Stabilized DDFV schemes for Stokes problem with variable viscosity on general $2 D$ meshes, Numer. Methods Partial Differential Equations, 27 (2011), pp. 1666-1706.

[29] S. Krell and G. Manzini, The discrete duality finite volume method for Stokes equations on three-dimensional polyhedral meshes, SIAM J. Numer. Anal., 50 (2012), pp. 808-837.

[30] K. Lipnikov, G. Manzini, and M. Shashkov, Mimetic finite difference method, J. Comput. Phys., 257 (2014), pp. 1163-1227.

[31] R. Rannacher and S. TureK, Simple nonconforming quadrilateral Stokes element, Numer. Methods Partial Differential Equations, 8 (1992), pp. 97-111.

[32] G. SAVARÉ, Regularity results for elliptic equations in Lipschitz domains, J. Funct. Anal., 152 (1998), pp. 176-201.

[33] A. Tabarraei and N. Sukumar, Conforming polygonal finite elements, Internat. J. Numer. Methods Engrg., 61 (2004), pp. 2045-2066.

[34] A. Tabarraei And N. Sukumar, Extended finite element method on polygonal and quadtree meshes, Comput. Methods Appl. Mech. Engrg., 197 (2007), pp. 425-438.

[35] C. Talischi, G. H. Paulino, A. Pereira, and I. F. M. Menezes, Polygonal finite elements for topology optimization: A unifying paradigm, Internat. J. Numer. Methods Engrg., 82 (2010), pp. 671-698.

[36] C. Talischi, G. H. Paulino, A. Pereira, and I. F. M. Menezes, PolyTop: A Matlab implementation of a general topology optimization framework using unstructured polygonal finite element meshes, J. Struct. Mult. Optim., 45 (2012), pp. 329-357.

[37] J. Wang, Y. WANG, AND X. Ye, A robust numerical method for Stokes equations based on divergence-free H(div) finite element methods, SIAM J. Sci. Comput., 31 (2009), pp. 27842802.

[38] P. Wesseling, Principles of Computational Fluid Dynamics, Springer Ser. Comput. Math. 29, Springer-Verlag, Berlin, 2001.

Copyright $@$ by SIAM. Unauthorized reproduction of this article is prohibited. 